\title{
Relationship between Body Mass Index and Coronary
}

\section{Atherosclerosis Analyzed by Multivessel Angioscopic Study}

\author{
Osamu Kurihara, MD, PhD, ${ }^{1}$ Masamichi Takano, $\mathrm{MD}, \mathrm{PhD},{ }^{* 1}$ Kyoichi Mizuno, MD, $\mathrm{PhD},{ }^{2}$ \\ Yusaku Shibata, MD, ${ }^{1}$ Masato Matsushita, MD, ${ }^{1}$ Hidenori Komiyama, MD, PhD, ${ }^{1}$ Katsuhito Kato, MD, PhD, ${ }^{1}$ \\ Ryo Munakata, MD, PhD, ${ }^{1}$ Daisuke Murakami, MD, PhD, ${ }^{1}$ Kentaro Okamatsu, MD, PhD, ${ }^{1}$ \\ Yasushi Miyauchi, MD, PhD, ${ }^{1}$ Noritake Hata, MD, PhD, ${ }^{1}$ Yoshihiko Seino, MD, PhD, ${ }^{1}$ \\ and Wataru Shimizu MD, PhD, ${ }^{3}$ \\ ${ }^{1}$ Cardiovascular Center, Chiba Hokusoh Hospital, Nippon Medical School, Chiba, Japan \\ ${ }^{2}$ Mitsukoshi Health and Welfare Foundation, Tokyo, Japan \\ ${ }^{3}$ Division of Cardiology, Nippon Medical School, Tokyo, Japan
}

Purpose: Low body mass index (BMI) is strongly associated with an increased long-term risk of cardiovascular events. However, there is no evidence of relationship between BMI and the degree of coronary atherosclerosis, and we evaluated the relationship using multivessel angioscopic observation.

Methods: A total of 89 patients with coronary artery disease who underwent angioscopic examination were analyzed. The patients were divided into four categories according to BMI levels: underweight: $<20 \mathrm{~kg} / \mathrm{m}^{2}(\mathrm{n}=7)$; normal weight: 20 to $<25 \mathrm{~kg} / \mathrm{m}^{2}(\mathrm{n}=40)$; overweight: 25 to $<28 \mathrm{~kg} / \mathrm{m}^{2}(\mathrm{n}=27)$; and obese: $>28 \mathrm{~kg} / \mathrm{m}^{2}(\mathrm{n}=15)$. The number of yellow plaques (NYP) through the observed coronary artery was counted and the color of each plaque was defined as grade 1 (light yellow), grade 2 (yellow), or grade 3 (intense yellow). The NYP per vessel and the maximum yellow grade (MYG) were compared between the four different groups of BMI.

Results: The severity of coronary atherosclerosis was significantly different: NYP per vessel ( $2.10 \pm 1.16$ vs. 1.53 \pm 0.91 vs. $1.33 \pm 0.79$ vs. $1.10 \pm 0.93, \mathrm{p}=0.024)$ and $\mathrm{MYG}(2.57 \pm 0.54$ vs. $2.25 \pm 0.87$ vs. $1.96 \pm 0.90$ vs. $1.93 \pm$ $1.03, \mathrm{p}=0.046)$. Multivariate regression analysis showed that BMI $(\mathrm{p}=0.024)$ independently correlate with the NYP per vessel.

Conclusions: Coronary atherosclerosis in underweight patients was more advanced than normal and overweight patients. Low BMI was independently related to progressive coronary atherosclerosis and that might be a characteristic of vulnerable patients.

Key words: body mass index, coronary artery disease, yellow plaque, obesity paradox

\section{Introduction}

Body mass index (BMI) is a common index used for obesity in both clinical practice and epidemiological studies, ${ }^{1)}$ and several studies have reported an inverse association between BMI and mortality in subjects with coronary artery disease (CAD), socalled the "obesity paradox." ${ }^{2,3}$

A meta-analysis of 40 studies showed that low BMI is strongly associated with an increased long-term risk of cardiovascular events; therefore, we hypothesized that low BMI may be a risk

\footnotetext{
* 1715 Kamakari, Inzai, Chiba, 270-1694 Japan

Email: takanom@nms.ac.jp

(Received 2016 03.05; Accepted 2016.08.19)

doi.org/10.15791/angioscopy.oa.16.0010
}

of coronary atherosclerosis. Coronary angioscopy is a robust imaging modality for assessment of atherosclerotic plaques in vivo. ${ }^{4-10)}$ Angioscopic yellow plaques are commonly observed at the culprit lesion in acute coronary syndrome (ACS), ${ }^{4-6)}$ and they are considered to be vulnerable. ${ }^{11)}$ In addition, the existence of multiple $(n \geq 2)$ yellow plaques in the single coronary trunk is regarded as a strong risk factor of future ACS events. ${ }^{12)}$ The aim of this study was to investigate the obesity paradox by multivessel angioscopic analysis.

\section{Methods}

\section{Patient population}

Our institution has a database of angioscopic analysis at the beginning of introducing this technology. Using this system, we 
enrolled all 89 CAD patients who underwent multivessel angioscopic observation between September 2000 and July 2007. ACS was defined as the occurrence of ischemic chest discomfort at rest with electrocardiography (ECG) changes specific to myocardial ischemia, regional wall motion abnormalities by echocardiography, or a previous history of CAD. Patients with elevated cardiac troponin T levels $(>0.01$ $\mathrm{ng} / \mathrm{ml}$ ) on admission or in the samples taken at 6-8 $\mathrm{h}$ after admission were diagnosed with acute myocardial infarction. No elevation of cardiac troponin $\mathrm{T}$ is diagnosed as unstable angina. Other patients were defined as stable CAD. Hypercholesterolemia was defined as receiving medication of hypercholesterolemia, fasting serum low-density lipoprotein cholesterol (LDL-C) $\geq$ $140 \mathrm{mg} / \mathrm{dl}$, or fasting serum total cholesterol $\geq 220 \mathrm{mg} / \mathrm{dl}$. CAD risk factor was defined as hypertension, hypercholesterolemia, diabetes, and smoking. The clinical and laboratory data were collected on the basis of the patients' charts. BMI is defined as the body weight divided by length in meters squared.

The patients were divided into four different BMI categories: $<20 \mathrm{~kg} / \mathrm{m}^{2}$ (underweight); 20 to $<25 \mathrm{~kg} / \mathrm{m}^{2}$ (normal weight); 25 to $<28 \mathrm{~kg} / \mathrm{m}^{2}$ (overweight); $\geq 28 \mathrm{~kg} / \mathrm{m}^{2}$ (obese), according to previous systematic review of cohort studies indicating the association of BMI and cardiovascular events.2)

\section{Angioscopic imaging and analysis}

The coronary angioscopic procedure has been reported previously. ${ }^{4-10)}$ Coronary arteries with proximal tortuosity, sharp angle, calcification, or ostial stenosis were not performed because of the expected difficulty in acquiring angioscopic images for the entire vessel. Before observation, the white balance was adjusted for color correction. The light power was adjusted to avoid reflection and to obtain images with adequate brightness for determination of the plaque color. During angioscopic observations, an assistant adjusted the light power to maintain a constant brightness level on the target plaque. Angioscopic images and fluoroscopy during the angioscopic observations were recorded simultaneously on digital videotape for later analysis. The total number of yellow plaques (NYP) was counted for each vessel. The yellow grade was classified semi-quantitatively according to the surface color as 0 , white; 1 , light yellow; 2, yellow; and 3, intense yellow. The maximum yellow grade (MYG) was defined as the highest yellow grade in all the coronary arteries for each patient. A ruptured plaque was considered to be a wall irregularity, including a tear, flap, or ulceration on the plaque surface. A thrombus was defined as a coalescent red superficial or protruding mass adhering to the vessel surface, but clearly a separate structure that remained after flushing with Ringer's lactate. The angioscopic images were evaluated by an investigator who was blinded to patients' clinical characteristics. In the previous study performed in our institution, the intra- and inter-observer agreements for evaluated angioscopic items (thrombus, yellow plaque, and complex plaque) were $95 \%, 100 \%$, and $95 \%$, respectively, and $95 \%, 98 \%$, and $93 \%$, respectively. The $\kappa$ values for the intraand inter-observer agreement of those items were $0.93,1.00$, and 0.88 , respectively, and $0.93,0.95$, and 0.84 , respectively. ${ }^{6}$ ) The medical ethics committee at Nippon Medical School Chiba Hokusoh Hospital approved this study protocol (no. 482), and written informed consent was obtained from all the patients before the catheterization procedures.

\section{Statistical analysis}

Continuous quantitative data were presented as mean value \pm standard deviation (SD). Comparisons between the four groups were done using analysis of variance (ANOVA) and the least significant difference statistical method. The JonckheereTerpstra trend test was used to test for ordered differences in the NYP per vessel and the MYG among the four groups. Categorical variables presented as frequencies were done with Pearson's chi-square test and the Holm method.

Simple linear regression analysis was applied to study the relationship between BMI and NYP per vessel, and multivariate regression analysis was done adjusting for confounding factors (i.e., age and gender) and coronary risk factors (i.e., prevalence of hypertension, smoking status, serum LDL-C, serum hemoglobin A1c (HbA1c), and incidence of ACS). All differences were evaluated at the $95 \%$ level of significance $(\mathrm{p}<$ 0.05). All statistical analyses were performed using IBM SPSS software version 21 (IBM Japan, Tokyo, Japan).

\section{Results}

\section{Patients' characteristics}

The baseline clinical characteristics are summarized in Table 1. Seven patients $(8 \%)$ were classified into underweight, 40 patients (45\%) into normal weight, 27 patients (30\%) into overweight, and 15 patients (17\%) into obese. Coronary risk factors including the frequencies of diabetes, hypercholesterolemia, hypertension, smoking status, and the other clinical presentations did not differ among the four groups, excepting for age and BMI levels. Age was higher in patients with underweight, normal weight, and over weight than obese (underweight vs. obese; $\mathrm{p}<$ 0.01 , normal weight vs. obese; $\mathrm{p}<0.001$, over weight vs. obese; $\mathrm{p}<0.05)$.

\section{Angioscopic findings}

The angioscopic findings are shown in Table 2. The distribution 
Body Mass Index and Coronary Atherosclerosis

Table 1. Patients' characteristics

\begin{tabular}{|c|c|c|c|c|c|}
\hline Patients, $\mathrm{n}$ & $\begin{array}{c}\text { Underweight } \\
7 \\
\end{array}$ & $\begin{array}{c}\text { Normal weight } \\
40\end{array}$ & $\begin{array}{c}\text { Overweight } \\
27\end{array}$ & $\begin{array}{c}\text { Obese } \\
15 \\
\end{array}$ & $\mathrm{p}$ Value \\
\hline Age, years & $65.4 \pm 8.1$ & $64.1 \pm 10.5$ & $59.3 \pm 8.6$ & $52.3 \pm 12.6$ & 0.002 \\
\hline Gender, male & $5(71 \%)$ & $29(73 \%)$ & $24(89 \%)$ & $13(87 \%)$ & 0.33 \\
\hline \multicolumn{6}{|l|}{$\mathrm{CAD}$ risk factor } \\
\hline Hypertension & $6(86 \%)$ & $21(53 \%)$ & $18(67 \%)$ & $12(80 \%)$ & 0.14 \\
\hline Hypercholesterolemia & $5(71 \%)$ & $37(93 \%)$ & $24(89 \%)$ & $14(93 \%)$ & 0.37 \\
\hline Diabetes & $2(29 \%)$ & $12(30 \%)$ & $7(26 \%)$ & $6(40 \%)$ & 0.82 \\
\hline Smoking & $3(43 \%)$ & $16(40 \%)$ & $7(26 \%)$ & $9(60 \%)$ & 0.19 \\
\hline \multicolumn{6}{|l|}{ Number of CAD risk factor } \\
\hline 0 & $0(0 \%)$ & $0(0 \%)$ & $0(0 \%)$ & $1(7 \%)$ & 0.307 \\
\hline 1 & $2(29 \%)$ & $8(20 \%)$ & $8(30 \%)$ & $1(7 \%)$ & \\
\hline 2 & $4(57 \%)$ & $19(48 \%)$ & $12(44 \%)$ & $4(26 \%)$ & \\
\hline 3 & $1(14 \%)$ & $10(25 \%)$ & $5(19 \%)$ & $6(40 \%)$ & \\
\hline 4 & $0(0 \%)$ & $3(7 \%)$ & $2(7 \%)$ & $3(20 \%)$ & \\
\hline Average number of CAD risk factor & $1.9 \pm 0.7$ & $2.2 \pm 0.9$ & $2.0 \pm 0.9$ & $2.6 \pm 1.1$ & 0.196 \\
\hline BMI $\left(\mathrm{kg} / \mathrm{m}^{2}\right)$ & $18.7 \pm 0.6$ & $22.7 \pm 1.4$ & $26.1 \pm 0.8$ & $30.0 \pm 2.1$ & $<0.001$ \\
\hline \multicolumn{6}{|l|}{ Clinical presentation } \\
\hline Acute coronary syndrome & $3(43 \%)$ & $16(40 \%)$ & $7(26 \%)$ & $7(47 \%)$ & 0.517 \\
\hline Acute MI & $3(43 \%)$ & $11(40 \%)$ & $4(26 \%)$ & $4(47 \%)$ & 0.414 \\
\hline UA & $0(0 \%)$ & $5(53 \%)$ & $3(74 \%)$ & $3(33 \%)$ & 0.607 \\
\hline Stable CAD & $4(57 \%)$ & $24(60 \%)$ & $20(74 \%)$ & $8(53 \%)$ & \\
\hline \multicolumn{6}{|l|}{ Medication } \\
\hline Statin & $4(57 \%)$ & $23(58 \%)$ & $17(63 \%)$ & $9(60 \%)$ & 0.93 \\
\hline ACE-I & $2(29 \%)$ & $11(28 \%)$ & $3(11 \%)$ & $3(20 \%)$ & 0.46 \\
\hline $\mathrm{ARB}$ & $2(29 \%)$ & $14(35 \%)$ & $11(41 \%)$ & $7(47 \%)$ & 0.78 \\
\hline Beta blockers & $3(43 \%)$ & $9(23 \%)$ & $9(33 \%)$ & $8(53 \%)$ & 0.16 \\
\hline Calcium channel blocker & $2(29 \%)$ & $11(28 \%)$ & $12(44 \%)$ & $8(53 \%)$ & 0.22 \\
\hline Dual-antiplatelet therapy & $6(86 \%)$ & $28(70 \%)$ & $18(67 \%)$ & $11(73 \%)$ & 0.84 \\
\hline \multicolumn{6}{|l|}{ Laboratory data } \\
\hline Total cholesterol (mg/dl) & $200.1 \pm 35.7$ & $197.2 \pm 33.8$ & $197.2 \pm 36.5$ & $197.0 \pm 31.3$ & 0.997 \\
\hline LDL-C (mg/dl) & $132.1 \pm 34.7$ & $121.7 \pm 33.6$ & $125.6 \pm 37.4$ & $113.3 \pm 35.9$ & 0.623 \\
\hline $\mathrm{HDL}-\mathrm{C}(\mathrm{mg} / \mathrm{dl})$ & $43.6 \pm 9.8$ & $49.6 \pm 16.7$ & $45.0 \pm 8.6$ & $42.9 \pm 10.2$ & 0.276 \\
\hline LDL/HDL ratio & $3.15 \pm 1.04$ & $2.67 \pm 1.21$ & $2.91 \pm 1.16$ & $2.76 \pm 1.07$ & 0.708 \\
\hline Triglyceride (mg/dl) & $120.7 \pm 38.5$ & $137.7 \pm 78.8$ & $133.0 \pm 52.2$ & $166.6 \pm 78.7$ & 0.387 \\
\hline $\mathrm{CRP}(\mathrm{mg} / \mathrm{dl})$ & $0.33 \pm 0.30$ & $0.26 \pm 0.46$ & $0.21 \pm 0.27$ & $0.46 \pm 0.99$ & 0.545 \\
\hline $\operatorname{HbA1c}(\%)$ & $6.0 \pm 2.0$ & $5.5 \pm 1.0$ & $5.8 \pm 1.3$ & $5.9 \pm 1.0$ & 0.526 \\
\hline
\end{tabular}

Values are numbers $(\%)$ or means \pm SD.

CAD: coronary artery disease, BMI: body mass index, MI: myocardial infarction, UA: unstable angina pectoris, ACE-I: angiotensin-converting enzyme inhibitors, ARB: angiotensin receptor blockers, LDL-C: low-density lipoprotein cholesterol, HDL-C: high-density lipoprotein cholesterol, CRP: C-reactive protein, HbA1c: hemoglobin A1c

Table 2. Angioscopic findings

\begin{tabular}{|c|c|c|c|c|c|}
\hline Patients, $\mathrm{n}$ & $\begin{array}{c}\text { Underweight } \\
7 \\
\end{array}$ & $\begin{array}{c}\text { Normal weight } \\
40\end{array}$ & $\begin{array}{c}\text { Overweight } \\
27 \\
\end{array}$ & $\begin{array}{c}\text { Obese } \\
15\end{array}$ & $\mathrm{p}$ Value \\
\hline \multicolumn{6}{|l|}{ Observed vessel } \\
\hline LAD & $5(71 \%)$ & $37(93 \%)$ & $25(93 \%)$ & $15(100 \%)$ & 0.142 \\
\hline $\mathrm{LCx}$ & $4(57 \%)$ & $29(73 \%)$ & $22(81 \%)$ & $9(60 \%)$ & 0.385 \\
\hline RCA & $6(86 \%)$ & $22(55 \%)$ & $16(59 \%)$ & $12(80 \%)$ & 0.198 \\
\hline Total NYP & 31 & 136 & 84 & 38 & \\
\hline NYP per patients & 4.4 & 3.4 & 3.1 & 2.5 & \\
\hline \multicolumn{6}{|l|}{ Yellow grade } \\
\hline Grade 1 & $10(32 \%)$ & $44(32 \%)$ & $37(44 \%)$ & $11(42 \%)$ & 0.150 \\
\hline Grade 2 & $12(39 \%)$ & $65(48 \%)$ & $39(46 \%)$ & $19(44 \%)$ & \\
\hline Grade 3 & $9(29 \%)$ & $27(20 \%)$ & $8(10 \%)$ & $8(14 \%)$ & \\
\hline Rupture, n & $2(29 \%)$ & $9(23 \%)$ & $3(11 \%)$ & $2(13 \%)$ & 0.538 \\
\hline Thrombus, $\mathrm{n}$ & $4(57 \%)$ & $13(33 \%)$ & $9(33 \%)$ & $6(40 \%)$ & 0.624 \\
\hline
\end{tabular}

Values are numbers (\%).

LAD: left anterior descending artery, LCx: left circumflex artery, RCA: right coronary artery, NYP: number of yellow plaques 
Table 3. Multivariate regression analysis for factors influencing number of yellow plaque per vessel

\begin{tabular}{|c|c|c|c|}
\hline Variables & $\beta$ Coefficient & $t$ Ratio & $p$ Value \\
\hline \multicolumn{4}{|l|}{ Clinical characteristics } \\
\hline Age (per 1-year increment) & 0.066 & 0.570 & 0.570 \\
\hline Gender (female) & 0.230 & 0.421 & 0.675 \\
\hline Hypertension & 0.160 & 1.511 & 0.135 \\
\hline Current smoking & -0.095 & -0.955 & 0.343 \\
\hline \multicolumn{4}{|l|}{ Clinical presentation } \\
\hline ACS & -0.043 & -0.427 & 0.670 \\
\hline BMI (per $0.1-\mathrm{kg} / \mathrm{m}^{2}$ decrement) & -0.256 & -2.308 & 0.024 \\
\hline \multicolumn{4}{|l|}{ Laboratory data } \\
\hline LDL-C (per 1-mg increment) & 0.339 & 3.219 & 0.002 \\
\hline HbA1c (per $0.1-\%$ increment) & 0.319 & 3.198 & 0.002 \\
\hline
\end{tabular}

ACS: acute coronary syndrome, BMI: body mass index, LDL-C: low-density lipoprotein, HbA1c: hemoglobin A1c

A. Number of Yellow Plaque per Vessel

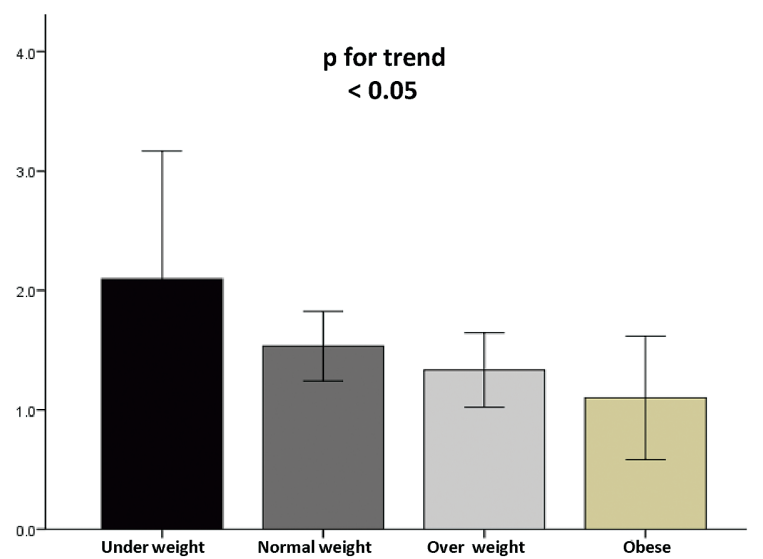

B. Maximum Yellow Grade

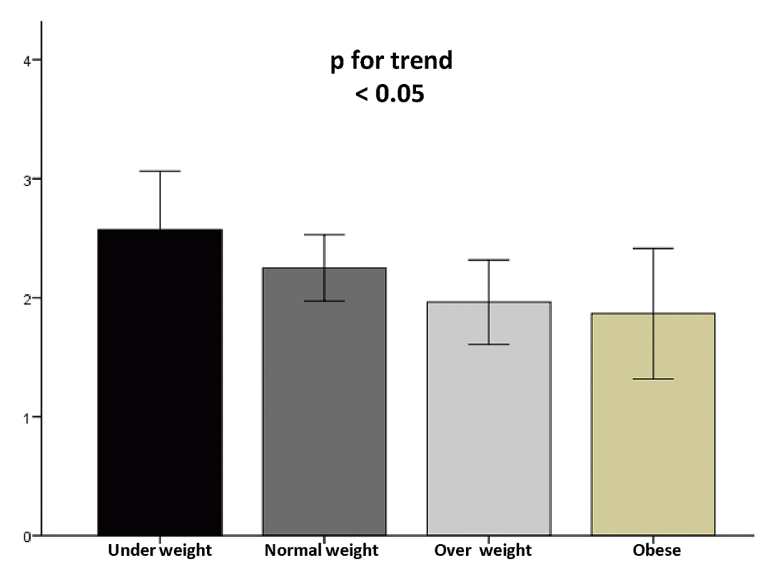

Fig. 1 Angioscopic comparisons of coronary atherosclerosis. The NYP per vessel (A) and MYG (B) are shown. The graded regression of the BMI categories was associated with the NYP per vessel ( $p=0.024$ for trend) and MYG ( $p=0.046$ for trend). NYP: number of yellow plaques, MYG: maximum yellow grade, BMI: body mass index

of the coronary arteries was observed, and the frequency of plaque rupture and thrombus were similar among the four groups. Figure 1 shows the relationship between BMI and angioscopic indexes of yellow plaque. The NYP per vessel and the MYG differed among the four groups $(2.10 \pm 1.16$ vs. $1.53 \pm 0.91$ vs. $1.33 \pm 0.79$ vs. $1.10 \pm 0.93, \mathrm{p}=0.024$, and $2.57 \pm 0.54$ vs. $2.25 \pm$ 0.87 vs. $1.96 \pm 0.90$ vs. $1.93 \pm 1.03, \mathrm{p}=0.046$, respectively).

On simple linear regression analysis, BMI inversely correlated with the NYP per vessel $(\mathrm{R}=-0.275, \mathrm{p}=0.009)$. Multivariate regression analysis showed, taking into account the predefined variables, BMI, serum LDL-C, and HbA1c level to independently correlate with the NYP per vessel (Table 3).

\section{Discussion}

This study showed that the NYP per vessel and MYG as angioscopic indexes of coronary atherosclerosis gradually increased according to decreasing in BMI.

Yellow plaque composed of a lipid pool or necrotic core under a thin fibrous cap is commonly found in the culprit lesion of ACS and that is considered vulnerable plaque..$^{4-6,13,14)}$ The yellow grade of the plaque is conversely correlated with fibrous cap thickness, and the thickness of fibrous cap covering of highgrade yellow plaque is extremely less. ${ }^{8,15,16)}$ Therefore, it is believed that high-grade yellow plaque is structurally fragile. In addition, angioscopic investigations reported that the patients with the existence of intense yellow plaque or multiple yellow plaques $(n \geq 2)$ per vessel is a high risk of suffering from ACS, and those are characteristics of vulnerable patients. ${ }^{12,15)}$ Therefore, both NYP per vessel and high MYG indicate not only the severity of coronary atherosclerosis but also patient's vulnerability.

There are many reports showing an inverse correlation between BMI and mortality in patients with CAD. ${ }^{3,17-19)}$ Although low $\mathrm{BMI}$ is associated with malignancy causing weight loss, the analysis involving 89,056 individuals registered with the Taiwan National Health Insurance showed that increased risk of non- 
cancer-related death was associated with low BMI, ${ }^{20)}$ and then low BMI is strongly associated with not only total mortality but also cardiovascular mortality. ${ }^{2)}$ For the first time, this angioscopic investigation directly revealed the association between decreasing BMI and advanced coronary atherosclerosis. However, we cannot clearly explain the relationship between low BMI and advanced coronary atherosclerosis. The several mechanisms of the relationship between BMI and cardiovascular events are considered. Some investigations reported that low lean mass is disadvantage to the incidence of cardiovascular events resulting from a restricted exercise capacity. ${ }^{21)}$ Cardiac rehabilitation using physical exercise shows marked reduction of plasma lipid levels and inflammation ${ }^{22}$ that might have inhibitory effect of atherosclerosis.

On the other hand, BMI is insufficient to discriminate between fat and lean mass, and unsuitable for estimating those distributions and obesity. ${ }^{23)}$ In a recent cohort study, low body fat and low lean mass evaluated without BMI invite high mortality of the patients. ${ }^{24)} \mathrm{BMI}$ is not a direct index of obesity; our findings therefore may mean not obesity paradox but BMI paradox. Regarding the relationship between BMI and cardiovascular events, prognosis of underweight and severe obesity is poorer than other groups, so-called U-shaped curve is found according to a previous meta-analysis. ${ }^{2)}$ However, subject of our study did not contain patients of severe obesity (BMI $\geq 35$ $\mathrm{kg} / \mathrm{m}^{2}$ ). As a result, our study did not show U-shaped curve.

Although BMI paradox in patients with CAD has been established, further researches are needed on the mechanism of BMI paradox. The relationship between lean mass or fatness and coronary atherosclerosis is required to determine the optimal $\mathrm{BMI}$ for prevention of CAD.

\section{Conclusions}

This study showed that coronary atherosclerosis gradually advanced according to decreasing in BMI. Low BMI was independently related to progressive coronary atherosclerosis and that might be a characteristic of vulnerable patients as well as previous studies.

\section{Study limitations}

The present findings were based on observations in a relatively limited number of patients, and excluded any patients who had coronary arteries that were not anatomically suitable for angioscopy (e.g., ostial stenotic, proximally tortuous, or severely calcified coronary arteries). Therefore, the present data should be considered as exploratory and hypothesis generating. The degree of atherosclerosis evaluated in this study might be higher than those in general because all patients had already some CAD at baseline.

\section{Acknowledgments}

None of the authors have any conflict of interest in relation to the research work described herein. No financial support was received for the study, nor do any of the authors have a relevant relationship with industry.

\section{References}

1) Pecorari D: [on 2 cases of partial obliteration of the uterine cavity]. Minerva Ginecol 1960; 12:1251-1255

2) Romero-Corral A, Montori VM, Somers VK, et al: Association of bodyweight with total mortality and with cardiovascular events in coronary artery disease: A systematic review of cohort studies. Lancet 2006; 368:666-678

3) Lavie CJ, Milani RV, Ventura HO: Obesity and cardiovascular disease: Risk factor, paradox, and impact of weight loss. J Am Coll Cardiol 2009; 53:1925-1932

4) Mizuno K, Miyamoto A, Satomura K, et al: Angioscopic coronary macromorphology in patients with acute coronary disorders. Lancet 1991; 337:809-812

5) Mizuno K, Satomura K, Miyamoto A, et al: Angioscopic evaluation of coronary-artery thrombi in acute coronary syndromes. N Engl J Med 1992; 326:287-291

6) Okamatsu K, Takano M, Sakai S, et al: Elevated troponin t levels and lesion characteristics in non-st-elevation acute coronary syndromes. Circulation 2004; 109:465-470

7) Takano M, Mizuno K, Yokoyama S, et al: Changes in coronary plaque color and morphology by lipid-lowering therapy with atorvastatin: Serial evaluation by coronary angioscopy. J Am Coll Cardiol 2003; 42:680-686

8) Takano M, Jang IK, Inami S, et al: In vivo comparison of optical coherence tomography and angioscopy for the evaluation of coronary plaque characteristics. Am J Cardiol 2008; 101:471476

9) Yamamoto M, Takano M, Okamatsu K, et al: Relationship between thin cap fibroatheroma identified by virtual histology and angioscopic yellow plaque in quantitative analysis with colorimetry. Circ J 2009; 73:497-502

10) Kurihara $O$, Takano $M$, Yamamoto $M$, et al: Impact of prediabetic status on coronary atherosclerosis: A multivessel angioscopic study. Diabetes Care 2013;36:729-733

11) Naghavi M, Libby P, Falk E, et al: From vulnerable plaque to vulnerable patient: A call for new definitions and risk assessment strategies: Part i. Circulation 2003; 108:1664-1672

12) Ohtani $T$, Ueda $Y$, Mizote I, et al: Number of yellow plaques detected in a coronary artery is associated with future risk of acute coronary syndrome: Detection of vulnerable patients by angioscopy. J Am Coll Cardiol 2006; 47:2194-2200

13) Sakai S, Mizuno K, Yokoyama S, et al: Morphologic changes in infarct-related plaque after coronary stent placement: A serial angioscopy study. J Am Coll Cardiol 2003; 42:1558-1565 
14) Takano M, Inami S, Ishibashi F, et al. Angioscopic follow-up study of coronary ruptured plaques in nonculprit lesions. J Am Coll Cardiol 2005; 45:652-658

15) Uchida $Y$, Nakamura F, Tomaru $T$, et al: Prediction of acute coronary syndromes by percutaneous coronary angioscopy in patients with stable angina. Am Heart J 1995; 130:195-203

16) Ishibashi F, Yokoyama S, Miyahara K, et al: Quantitative colorimetry of atherosclerotic plaque using the $\mathrm{l}^{*} \mathrm{a}^{*} \mathrm{~b} *$ color space during angioscopy for the detection of lipid cores underneath thin fibrous caps. Int J Cardiovasc Imaging. 2007; 23:679-691

17) Mehta L, Devlin W, McCullough PA, et al: Impact of body mass index on outcomes after percutaneous coronary intervention in patients with acute myocardial infarction. Am J Cardiol 2007; 99:906-910

18) Oreopoulos A, McAlister FA, Kalantar-Zadeh K, et al: The relationship between body mass index, treatment, and mortality in patients with established coronary artery disease: A report from approach. Eur Heart J 2009; 30:2584-2592

19) Angeras O, Albertsson P, Karason K, et al: Evidence for obesity paradox in patients with acute coronary syndromes: A report from the swedish coronary angiography and angioplasty registry. Eur Heart J 2013; 34:345-353

20) Dixon JB, Lambert GW. The obesity paradox: A reality that requires explanation and clinical interpretation. Atherosclerosis 2013; 226:47-48

21) Wallner $S$, Watzinger $N$, Lindschinger $M$, et al: Effects of intensified lifestyle modification on the need for further revascularization after coronary angioplasty. Eur J Clin Invest 1999; 29:372-379

22) Lavie CJ, Milani RV, Artham SM, et al: The obesity paradox, weight loss, and coronary disease. Am J Med 2009; 122:11061114

23) Romero-Corral A, Somers VK, Sierra-Johnson J, et al. Diagnostic performance of body mass index to detect obesity in patients with coronary artery disease. Eur Heart J 2007; 28:2087-2093

24) Lavie CJ, De Schutter A, Patel DA, et al: Body composition and survival in stable coronary heart disease: Impact of lean mass index and body fat in the "obesity paradox". J Am Coll Cardiol 2012; 60:1374-1380 